\title{
1/F NOISE AND TWO-LEVEL SYSTEMS IN JOSEPHSON QUBITS
}

Alexander Shnirman ${ }^{1}$, Gerd Schön ${ }^{1}$, Ivar Martin ${ }^{2}$, and Yuriy Makhlin ${ }^{3}$

${ }^{1}$ Institut für Theoretische Festkörperphysik, Universität Karlsruhe, D-76128 Karlsruhe, Germany

${ }^{2}$ Theoretical Division, Los Alamos National Laboratory, Los Alamos, NM 87545, USA

${ }^{3}$ Landau Institute for Theoretical Physics, Kosygin st. 2, 119334 Moscow, Russia

\begin{abstract}
Quantum state engineering in solid-state systems is one of the most rapidly developing areas of research. Solid-state building blocks of quantum computers have the advantages that they can be switched quickly, and they can be integrated into electronic control and measuring circuits. Substantial progress has been achieved with superconducting circuits (qubits) based on Josephson junctions. Strong coupling to the external circuits and other parts of the environment brings, together with the advantages, the problem of noise and, thus, decoherence. Therefore, the study of sources of decoherence is necessary. Josephson qubits themselves are very useful in this study: they have found their first application as sensitive spectrometers of the surrounding noise.
\end{abstract}

Key words: Josephson qubits, $1 / f$ noise

\section{Introduction}

Josephson junction based systems are one of the promising candidates for quantum state engineering with solid state systems. In recent years great progress was achieved in this area. After initial breakthroughs of the groups in Saclay and NEC (Tsukuba) in the late 90's, there are now many experimental groups worldwide working in this area, many of them with considerable previous experience in nano-electronics. By now the full scope of single-qubit (NMR-like) control is possible. One can drive Rabi oscillations, observe Ramsey fringes, apply composite pulses and echo technique (Collin et al., 2004). The goal of 'single shot' measurements has almost been achieved (Astafiev et al., 2004b; Siddiqi et al., 2004). There are first reports about 2-bit operations (Yamamoto et al., 2003). The decoherence times have reached microseconds, which would allow for hundreds of gates. Finally, a setup equivalent to cavity QED was realized in superconducting circuits (Wallraff et al., 2004). We refer the reader to the recent reviews (Esteve and Vion, 2005; Wendin and Shumeiko, 2005).

Despite the great progress decoherence remains the limiting factor in solid state circuits. Since one wants to manipulate and measure the qubits, some de- 


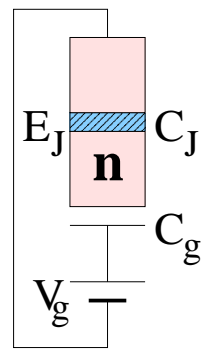

Figure 1. Charge Qubit.

coherence is unavoidable. There are, however, noise sources which are purely intrinsic, i.e., they are not related to any controlling or measuring circuitry. Eliminating those sources as much as possible is therefore of greatest importance. The main intrinsic source of decoherence in most superconducting qubits is $1 / f$ noise of either the charge, the flux or the critical Josephson current.

On the other hand, the full control of 1-qubit circuits opens the possibility to use qubits as efficient noise detectors (Aguado and Kouwenhoven, 2000; Schoelkopf et al., 2003). The idea is to measure the decoherence times of the qubit while changing its parameters and extract from the data the noise in the qubit's environment. An experiment of this type was performed by (Astafiev et al., 2004a). Further information about the noise was obtained in recent studies (Ithier et al., 2005; Martinis et al., 2005). In this paper we give a short overview of new and improved understanding of the nature of $1 / f$ noise.

\section{Charge qubit and charge noise}

To introduce the basic concepts we consider the simplest charge qubit. The system is shown in Fig. 1. Its Hamiltonian reads

$$
H=\sum_{n}\left[E_{\mathrm{ch}}\left(n, V_{\mathrm{g}}\right)|n\rangle\left\langle n\left|+\frac{E_{\mathrm{J}}}{2}\right| n\right\rangle\langle n \pm 1|\right],
$$

where the charging energy in given by

$$
E_{\mathrm{ch}}\left(n, V_{\mathrm{g}}\right)=\frac{\left(2 n e-Q_{\mathrm{g}}\right)^{2}}{2\left(C_{\mathrm{g}}+C_{\mathrm{J}}\right)},
$$

and the induced gate charge is $Q_{\mathrm{g}}=C_{\mathrm{g}} V_{\mathrm{g}}$. Near $Q_{\mathrm{g}}=e$ one can consider the two lowest energy charge states. In the spin- $1 / 2$ representation one obtains the following Hamiltonian

$$
H=-\frac{1}{2} \Delta E_{\mathrm{ch}}\left(V_{\mathrm{g}}\right) \hat{\sigma}_{z}-\frac{1}{2} E_{\mathrm{J}} \hat{\sigma}_{x},
$$


Introducing an angle $\eta\left(V_{\mathrm{g}}\right)$ such that $\tan \eta=E_{\mathrm{J}} / E_{\mathrm{ch}}\left(V_{\mathrm{g}}\right)$ we rewrite the Hamiltonian as

$$
H=-\frac{1}{2} \Delta E\left(\cos \eta \hat{\sigma}_{z}+\sin \eta \hat{\sigma}_{x}\right) .
$$

We now assume that the gate charge has a noisy component, i.e., $Q_{\mathrm{g}}=C_{\mathrm{g}} V_{\mathrm{g}}+\delta Q$. Then the charging energy fluctuates and we obtain

$$
H=-\frac{1}{2} \Delta E\left(\cos \eta \hat{\sigma}_{z}+\sin \eta \hat{\sigma}_{x}\right)-\frac{1}{2} X \hat{\sigma}_{z},
$$

where $X=e \delta Q /\left(C_{\mathrm{g}}+C_{\mathrm{J}}\right)$. In the eigenbasis of the qubit this gives

$$
H=-\frac{1}{2} \Delta E \hat{\sigma}_{z}-\frac{1}{2} X\left(\cos \eta \hat{\sigma}_{z}-\sin \eta \hat{\sigma}_{x}\right) .
$$

For sufficiently weak noise with regular spectrum $S_{X}(\omega)$, the Bloch-Redfield theory (Bloch, 1957; Redfield, 1957) gives the dissipative rates. The relaxation (spin flip) rate is given by

$$
\Gamma_{1} \equiv \frac{1}{T_{1}}=\frac{1}{2} \sin ^{2} \eta S_{X}(\omega=\Delta E),
$$

while the dephasing rate

$$
\Gamma_{2} \equiv \frac{1}{T_{2}}=\frac{1}{2} \Gamma_{1}+\Gamma_{\varphi}
$$

with

$$
\Gamma_{\varphi}=\frac{1}{2} \cos ^{2} \eta S_{X}(\omega=0) .
$$

is a combination of spin-flip effects $\left(\Gamma_{1}\right)$ and of the so called 'pure' dephasing, characterized by the rate $\Gamma_{\varphi}=1 / T_{2}^{*}$. The pure dephasing is usually associated with the inhomogeneous level broadening in ensembles of spins, but occurs also for a single spin due to the 'longitudinal' (coupling to $\sigma_{z}$ ) low-frequency noise.

We now consider the situation where the noise $X$ is characterized by the spectral density

$$
S_{X}(\omega)=\frac{\alpha}{|\omega|}
$$

in the interval of frequencies $\omega_{\text {ir }}<\omega<\omega_{\mathrm{c}}$. In this case Eq. (9) is clearly inapplicable. Several models of $1 / f$ noise and pure dephasing were developed in the literature (Cottet, 2002; Shnirman et al., 2002; Paladino et al., 2002; Galperin et al., 2004a). In all of them the $T_{2}$-decay of the coherences (i.e. of the offdiagonal elements of the density matrix) is given by decay law $e^{-\Gamma_{1} t / 2} f(t)$. The pure decoherence described by the function $f(t)$ depends on the statistics of the noise. For our purposes here a very rough estimate is enough. When deriving the 


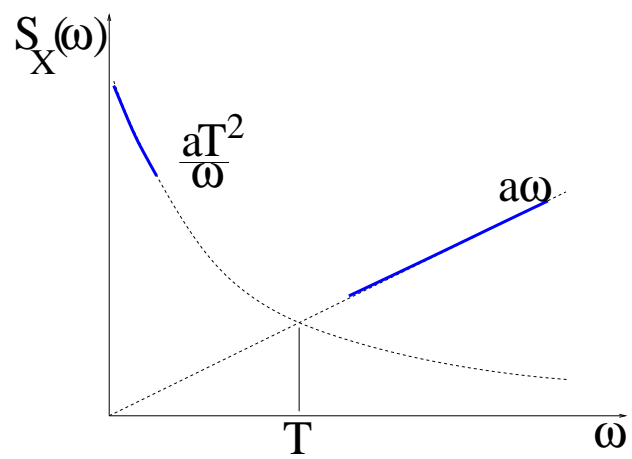

Figure 2. Asymptotic behavior of noise at low and high frequencies.

Bloch-Redfield results, e.g., Eq. (9), one realizes that $S(\omega=0)$ should actually be understood as the noise power averaged over the frequency band of width $\sim \Gamma_{\varphi}$ around $\omega=0$. We, thus, obtain a time scale of the pure dephasing from the self-consistency condition $\Gamma_{\varphi}=S_{X}\left(\Gamma_{\varphi}\right)$. This gives

$$
\Gamma_{\varphi} \approx \sqrt{\alpha} \cos \eta
$$

For the cases of "strongly non-Gaussian" statistics (Galperin et al., 2004a), $\alpha$ and $\Gamma_{\varphi}$ should be understood as typical rather than ensemble averaged quantities. From the study of many examples we came to the conclusion that the relation (11) is universal irrespective of the noise statistics as long as $\Gamma_{\varphi}>\omega_{\mathrm{ir}}$.

\section{Analysis of the NEC experiments}

(Astafiev et al., 2004a) measured the $T_{1}$ and $T_{2}^{*}$ time scales in a charge qubit. As the energy splitting $\Delta E$ and the angle $\eta$ were independently controlled, they could extract the noise power $S(\omega)$ in the $\mathrm{GHz}$ range using Eq. (7). In addition they were able to determine the strength of the $1 / f$ noise, $\alpha$, using Eq. (11). The results suggested a connection between the strengths of the Ohmic high-frequency noise, responsible for the relaxation of the qubit ( $T_{1}$-decay), and the low-frequency $1 / f$ noise, which dominates the dephasing $\left(T_{2}\right.$-decay). The noise power spectra, extrapolated from the low- and high-frequency sides, turn out to cross at $\omega$ of order $T$. Expressing the high-frequency noise at $\omega>T$ as $S_{X}(\omega)=a \omega$, they found that the strength of the low-frequency noise scales as $\alpha=a T^{2}$ (see Fig. 2). The $T^{2}$ dependence of the low-frequency noise power was observed earlier for the $1 / f$ noise in Josephson devices (Wellstood, 1988; Kenyon et al., 2000). Further evidence for the $T^{2}$ behavior was obtained recently (Astafiev, 2004; Wellstood et al., 2004). But the fact that the two parts of the spectrum are characterized by the same constant $a$ was surprising. 


\section{Resonances in phase qubits}

Additional information was obtained from experiments with phase qubits (current biased large area Josephson junction) by (Simmonds et al., 2004). These experiments revealed the presence of spurious quantum two-level systems with strong effects on the high-frequency $(\sim 10 \mathrm{GHz})$ qubit dynamics. In a phase qubit one controls the energy splitting between the qubit states by changing the bias current. It turned out that at certain values of the bias current the system ceased to behave as a two-level system but showed rather a 4-level dynamics. This phenomenon can be attributed to the existence of a collection of coherent two-level fluctuators in the oxide of the tunnel barrier. When the energy splitting of the qubit coincides with that of one of the fluctuators a pair of states $\left|g_{\text {qubit }}\right\rangle\left|e_{\text {fluctuator }}\right\rangle$ and $\left|e_{\text {qubit }}\right\rangle\left|g_{\text {fluctuator }}\right\rangle$ are degenerate. Here $\left|g_{\text {qubit }}\right\rangle$ and $\left|g_{\text {fluctuator }}\right\rangle$ denote the ground states of the qubit and the fluctuator respectively, while $\left|e_{\text {qubit }}\right\rangle$ and $\left|e_{\text {fluctuator }}\right\rangle$ are the excited states. This degeneracy is lifted by the qubit-fluctuator interaction, which leads to a gap (avoided crossing) in the spectroscopy of the qubit. The most surprising was the observation that the two-level fluctuators are more coherent than the qubit. Hence, the decoherence of the 4-level system in a resonant situation is dominated by the decoherence of the qubit.

\section{High- and low-frequency noise from coherent TLF's}

Motivated by the above mentioned experiments we have pointed out (Shnirman et al., 2005) that a set of coherent two-level systems may produce both highand low-frequency noise with strengths that are naturally related. As a model we consider a set of coherent two-level systems described by the Pauli matrices $\sigma_{p, j}$, where $p=x, y, z$, and $j$ labels the particular TLF. We write the Hamiltonian of the set in the basis such that their contributions to the relevant fluctuating quantity, e.g., the gate charge, are $X \equiv \sum_{j} v_{j} \sigma_{z, j}$. Then

$$
H_{\mathrm{TLS}}=\sum_{j}\left[-\frac{1}{2}\left(\varepsilon_{j} \sigma_{z, j}+\Delta_{j} \sigma_{x, j}\right)+H_{\mathrm{diss}, j}\right] .
$$

Here, in the language of tunneling TLSs (TTLS), $\varepsilon_{j}$ are the bias energies and $\Delta_{j}$ the tunnel amplitudes between two states. Each individual TLS, $j$, is subject to dissipation due to its own bath with Hamiltonian $H_{\mathrm{diss}, j}$. We do not specify $H_{\mathrm{diss}, j}$, but only assume that it produces the usual relaxation $\left(T_{1}\right)$ and dephasing $\left(T_{2}\right)$ processes. We assume that all the TLSs are under-damped, with $\Gamma_{1, j} \equiv T_{1, j}^{-1} \ll E_{j}$ and $\Gamma_{2, j} \equiv T_{2, j}^{-1} \ll E_{j}$. Here $E_{j} \equiv \sqrt{\epsilon_{j}^{2}+\Delta_{j}^{2}}$ is the energy splitting. 
Our goal in the following is to investigate the noise properties of of the fluctuating field $X$. For that reason we evaluate the (unsymmetrized) correlator

$$
C_{X}(\omega) \equiv \int d t\left\{\langle X(t) X(0)\rangle-\langle X\rangle^{2}\right\} e^{i \omega t}
$$

For independent TLSs the noise is a sum of individual contributions, $C_{X}=\sum_{j} v_{j}^{2} C_{j}$, where

$$
C_{j}(\omega) \equiv \int d t\left\{\left\langle\sigma_{z, j}(t) \sigma_{z, j}(0)\right\rangle-\left\langle\sigma_{z, j}\right\rangle^{2}\right\} e^{i \omega t} .
$$

To obtain $C_{j}$ we first transform to the eigenbasis of the TLS. This gives

$$
H_{\mathrm{TLS}}=\sum_{j}\left\{-\frac{1}{2} E_{j} \rho_{z, j}+H_{\mathrm{diss}, j}\right\},
$$

and

$$
X=\sum_{j} v_{j}\left(\cos \theta_{j} \rho_{z, j}-\sin \theta_{j} \rho_{x, j}\right),
$$

where $\tan \theta_{j} \equiv \Delta_{j} / \epsilon_{j}$. Proceeding in the spirit of the Bloch-Redfield theory (Bloch, 1957; Redfield, 1957) we readily find

$$
\begin{aligned}
C_{j}(\omega) & \approx \cos ^{2} \theta_{j}\left[1-\left\langle\rho_{z, j}\right\rangle^{2}\right] \frac{2 \Gamma_{1, j}}{\Gamma_{1, j}^{2}+\omega^{2}} \\
& +\sin ^{2} \theta_{j}\left[\frac{1+\left\langle\rho_{z, j}\right\rangle}{2}\right] \frac{2 \Gamma_{2, j}}{\Gamma_{2, j}^{2}+\left(\omega-E_{j}\right)^{2}} \\
& +\sin ^{2} \theta_{j}\left[\frac{1-\left\langle\rho_{z, j}\right\rangle}{2}\right] \frac{2 \Gamma_{2, j}}{\Gamma_{2, j}^{2}+\left(\omega+E_{j}\right)^{2}} .
\end{aligned}
$$

In thermal equilibrium we have $\left\langle\rho_{z, j}\right\rangle=\tanh \left(E_{j} / 2 T\right)$. The first term, due to the longitudinal part of the coupling, describes random telegraph noise of a thermally excited TLS. We have assumed $\Gamma_{1, j} \ll T$, so that this term is symmetric (classical). The second term is due to the transverse coupling and describes absorption by the TLS, while the third term describes the transitions of the TLS with emission. We observe that TLSs with $E_{j} \gg T$ contribute to $C_{X}$ only at the (positive) frequency $\omega=E_{j}$. Indeed their contribution at $\omega=0$ is suppressed by the thermal factor $1-\left\langle\rho_{z, j}\right\rangle^{2}=1-\tanh ^{2}\left(E_{j} / 2 T\right)$. Also the negative frequency (emission) contribution at $\omega=-E_{j}$ is suppressed. These high-energy TLSs remain always in their ground state. Only the TLSs with $E_{j}<T$ are thermally excited, performing real random transitions between their two eigenstates, and contribute at $\omega= \pm E_{j}$ and at $\omega=0$. Note that the separation of the terms in Eq. (17) into low- and highfrequency noise is meaningful only provided the typical width $\Gamma_{1, j}$ of the low- $\omega$ 
Lorentzians is lower than the high frequencies of interest, which are defined, e.g., by the qubit's level splitting or temperature.

For a dense distribution of the parameters $\epsilon, \Delta$, and $v$ we can evaluate the lowand high-frequency noise. For positive high frequencies, $\omega \gg T$, we obtain

$$
\begin{aligned}
& C_{X}(\omega) \approx \sum_{j} v_{j}^{2} \sin ^{2} \theta_{j} \frac{2 \Gamma_{2, j}}{\Gamma_{2, j}^{2}+\left(\omega-E_{j}\right)^{2}} \\
& \approx N \int d \epsilon d \Delta d v P(\epsilon, \Delta, v) v^{2} \sin ^{2} \theta \cdot 2 \pi \delta(\omega-E),
\end{aligned}
$$

where $N$ is the number of fluctuators, $P(\epsilon, \Delta, v)$ is the distribution function normalized to $1, E \equiv \sqrt{\epsilon^{2}+\Delta^{2}}$, and $\tan \theta=\Delta / \epsilon$. Without loss of generality we take $\epsilon \geq 0$ and $\Delta \geq 0$.

At negative high frequencies $(\omega<0$ and $|\omega|>T)$ the correlator $C_{X}(\omega)$ is exponentially suppressed. On the other hand, the total weight of the low-frequency noise (up to $\omega \approx \Gamma_{1, \max }$, where $\Gamma_{1, \max }$ is the maximum relaxation rate of the TLSs) follows from the first term of (17). (Since we have assumed $\Gamma_{1, j} \ll E_{j}$ we can disregard the contribution of the last two terms of (17).) Each Lorentzian contributes 1 . Thus we obtain

$$
\begin{aligned}
& \int_{\text {low freq. }} \frac{d \omega}{2 \pi} C_{X}(\omega) \\
& \approx \int_{\text {low freq. }} \frac{d \omega}{2 \pi} \sum_{j} v_{j}^{2} \cos ^{2} \theta_{j}\left[1-\left\langle\rho_{z, j}\right\rangle^{2}\right] \frac{2 \Gamma_{1, j}}{\Gamma_{1, j}^{2}+\omega^{2}} \\
& \approx N \int d \epsilon d \Delta d v P(\epsilon, \Delta, v) v^{2} \cos ^{2} \theta \frac{1}{\cosh ^{2} \frac{E}{2 T}} .
\end{aligned}
$$

Equations (18) and (19) provide the general framework for further discussion.

Next we investigate possible distributions for the parameters $\epsilon, \Delta$, and $v$. We consider a log-uniform distribution of tunnel splittings $\Delta$, with density $P_{\Delta}(\Delta) \propto$ $1 / \Delta$ in a range $\left[\Delta_{\min }, \Delta_{\max }\right]$. This distribution is well known to provide for the $1 / f$ behavior of the low-frequency noise (Dutta and Horn, 1981a). It is natural for TTLSs as $\Delta$ is an exponential function of, e.g., the tunnel barrier height (Phillips, 1972), which is an almost uniformly distributed parameter. The relaxation rates are, then, also distributed log-uniformly, $P_{\Gamma_{1}}\left(\Gamma_{1}\right) \propto 1 / \Gamma_{1}$, and the sum of many Lorentzians of width $\Gamma_{1}$ centered at $\omega=0$ adds up to the $1 / f$ noise.

The distribution of $v$ is rather arbitrary. We only only assume that it is uncorrelated with $\varepsilon$ and $\Delta$. Finally we have to specify the distribution of $\epsilon \mathrm{s}$. First, 
we assume that the temperature is lower than $\Delta_{\max }$. For the high-frequency part, $T<\omega<\Delta_{\max }$, we find, after taking the integral over $\Delta$ in Eq. (18), that

$$
C_{X}(\omega) \propto \frac{1}{\omega} \int_{0}^{\omega} P_{\varepsilon}(\varepsilon) d \varepsilon .
$$

This is consistent with the observed Ohmic behavior $C_{X} \propto \omega$ only for a linear distribution $P_{\varepsilon}(\varepsilon) \propto \varepsilon$.

Remarkably, this distribution, $P(\varepsilon, \Delta) \propto \varepsilon / \Delta$, produces at the same time the $T^{2} \ln \left(T / \Delta_{\min }\right)$ behavior of the low-frequency weight (19), observed in several experiments (Wellstood, 1988; Kenyon et al., 2000; Astafiev, 2004; Wellstood et al., 2004). If the low-frequency noise has a $1 / f$ dependence, the two parts of the spectrum would cross around $\omega \sim T$ (Astafiev et al., 2004a).

In the opposite limit, $T \gg \Delta_{\max }$, the high-frequency noise depends on the detailed shape of the cutoff of $P_{\Delta}(\Delta)$ at $\Delta_{\max }$. As an example, for a hard cutoff the Ohmic spectral density implies that $P_{\varepsilon} \propto \varepsilon^{3}$, and the low-frequency weight scales with $T^{4}$. For a $1 / f$ low-frequency behavior, the spectra would cross at $\omega \sim$ $T^{2} / \Delta_{\max } \gg T$, which is not in agreement with the result of Ref. (Astafiev et al., 2004a).

A remark is in order concerning the crossing at $\omega \approx T$ discussed above. It is not guaranteed that the spectrum has a $1 / f$ dependence up to $\omega \sim T$. Rather the high-frequency cutoff of the low-frequency $1 / f$ noise is given by the maximum relaxation rate of the TLSs, $\Gamma_{1, \max } \ll T$, as we assumed. Then the extrapolations of the low-frequency $1 / f$ and high-frequency Ohmic spectra cross at this $\omega \sim T$.

We would like to emphasize that the relation between low- and high-frequency noise is more general, i.e., it is not unique to an ensemble of two-level systems. Consider an ensemble of many-level systems with levels $|n\rangle$ and energies $E_{n}$ such that the coupling is via an observable which has both transverse and longitudinal components. By 'transverse component' we mean the part constructed with operators $|n\rangle\langle m|$, where $n \neq m$, while the 'longitudinal component' is built from the projectors $|n\rangle\langle n|$. If the system is under-damped, that is, if the absorption and emission lines are well defined, the correlator of such an observable will have Lorentzian-like contributions at $\omega=E_{n}-E_{m}$ as well as at $\omega=0$. An example is provided by an ensemble of an-harmonic oscillators with $X=\sum_{j} v_{j} x_{j}$, where $x_{j}$ are the oscillator's coordinates. Due to the anharmonicity $x_{j}$ acquires a longitudinal component, in addition to the usual transverse one. Thus a relation between the low- and high-frequency noise would emerge naturally with details depending on the ensemble statistics. 


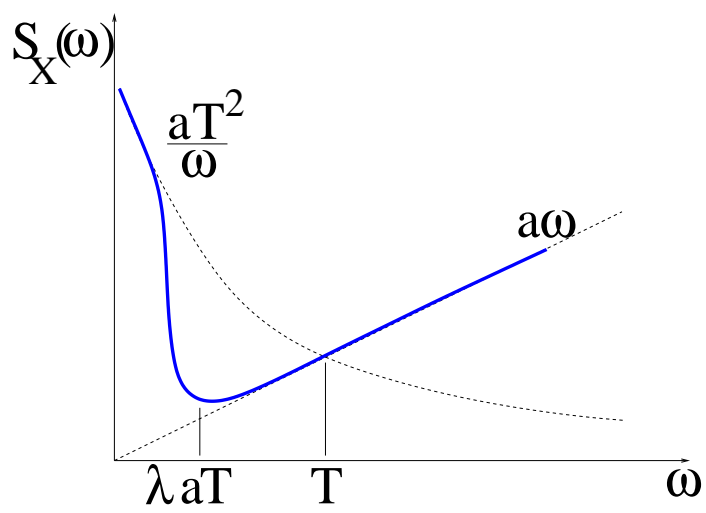

Figure 3. Noise spectrum in a selfconsistent model.

\section{Self-consistent model}

In this section we consider a possibility that the $\Gamma_{1}$ decay of each individual TLS is caused by the other TLSs. This model explains further details of the behavior of $S_{X}(\omega)$. We assume that each individual fluctuator "feels" the same charge noise as the qubit, however reduced by a factor $\lambda<1$ due to the small size of the fluctuators. That is we assume that the relaxation rate of the fluctuators is given by

$$
\Gamma_{1, j}=\frac{\lambda}{2} \sin \theta_{j}^{2} S_{X}\left(\omega=E_{j}\right) .
$$

As only the fluctuators with $E_{j} \leq T$ contribute to the $1 / f$ noise, we estimate the maximum possible relaxation rate of the fluctuators to be $\Gamma_{1, \max } \sim \lambda a T$. This leads to a crossover from $1 / f$ to $1 / f^{2}$ dependence around $\omega \sim \Gamma_{1, \max } \sim \lambda a T$ as indicated in Fig. 3. We note that such a crossover (soft cut-off) is compatible with the recent experimental data (Ithier et al., 2005).

\section{Relation to other work}

It is useful to relate our phenomenological results to the recent work of (Faoro et al., 2005), (de Sousa et al., 2005), (Grishin et al., 2005), and (Faoro and Ioffe, 2005), where physical models of the fluctuators, coupling to and relaxing the qubit, were considered. In Ref. (Faoro et al., 2005) three models were studied: (I) a single electron trap in tunnel contact with a metallic gate, (II) a single electron occupying a double trap, and (III) a double trap that can absorb/emit a Cooper pair from the qubit or a superconducting gate ('Andreev fluctuator'). In all models a uniform distribution of the trap energy levels was assumed. One, then, can show that the distribution for the two-level systems corresponding to the models II and III are linear in the energy level splitting, $P(\epsilon) \propto \epsilon$. Since the switching 
in these models is tunneling dominated, we find that $P(\Delta) \propto 1 / \Delta$. Therefore, both models II and III are characterized by distribution $P(\epsilon, \Delta) \propto \epsilon / \Delta$, introduced above, and hence can naturally account for the experimentally observed low- and high-frequency noises. In contrast, as shown in (de Sousa et al., 2005; Grishin et al., 2005), single electron traps do not behave as coherent two-level systems. Depending on the ratio between the hybridization with the metal and the temperature a single trap at the Fermi energy can either show the random telegraph noise or, when the hybridization dominates, it makes the qubit to feel the Ohmic particle-hole spectrum of the metal.

It was argued recently (Faoro and Ioffe, 2005) that one needs an unphysically high density of fluctuators in order to explain the experimental findings. This argument is based on an assumption that the traps' energies are distributed homogeneously over the energy band of order of the Fermi energy (of order $1 \mathrm{eV}$ ). (Faoro and Ioffe, 2005) proposed an alternative scenario where the low energy scale needed for qubit relaxation is provided by Kondo physics.

\section{Mechanisms of coupling between Josephson qubits and two-level systems}

In this section we discuss possible mechanisms of coupling between the TLS and the Josephson qubits in light of the recent experimental findings (Simmonds et al., 2004; Astafiev et al., 2004a; Martinis et al., 2005). For qubits with strong domination of the charging energy, like those of Ref. (Astafiev et al., 2004a), the charge noise considered in Section 2 is the main source of dissipation. In these qubits the fluctuators are most likely located away from the tunnel junction. This follows from the fact that a single charge of $e$ jumping back and forth a distance of a single atomic bond $(\sim 0.1 \mathrm{~nm})$ across a $2 \mathrm{~nm}$ wide tunnel junction (Martinis et al., 2005) would create charge noise with amplitude (defined as square root of integrated power) of order $10^{-2} \mathrm{e}$. A typical amplitude of the charge noise in single electron devices is smaller, $\sim 10^{-3} e$. If a fluctuator of this type would happen to be in the tunnel junction, such an "unfortunate" sample would, probably, be discarded by experimentalists. On the other hand, as we have already mentioned in Section 4, the coherent two-level systems have been observed in large area Josephson junctions. There are at least two types of interactions which could be responsible for lifting the degeneracy between the states $\left|g_{\text {qubit }}\right\rangle\left|e_{\text {fluctuator }}\right\rangle$ and $\left|e_{\text {qubit }}\right\rangle\left|g_{\text {fluctuator }}\right\rangle$ (Martin et al., 2005). One corresponds to a situation in which a two-level fluctuator blocks a conducting channel in one of its states and, thus, influences the Josephson energy of the junction (Simmonds et al., 2004). This can be expressed by substituting the Josephson energy as

$$
E_{J} \rightarrow E_{J}\left[1+\frac{1}{2} \sum_{j} v_{j} \sigma_{z, j}\right],
$$


where $v_{j}=\delta G_{j} / G$ is the ratio of the conductance variation $\delta G_{j}$ due to the motion of the fluctuator $j$ to the total tunnel conductance $G$ of the junction. The other coupling mechanism (Martin et al., 2005) arises due to a dipole moment of the fluctuator interacting with the electric field in the junction, i.e., it is directly related to the charge noise. The interaction energy of this mechanism can be written as $E_{\text {int }}=(1 / 2) e V \sum_{j} \tilde{v}_{j} \sigma_{z, j}$, where $V$ is the (operator of) voltage across the junction. The coupling constants $\tilde{v}_{j}$ are given this time by $\tilde{v}_{j}=d_{j} / l$, where $l$ is the width of the junction and $d_{j}$ is the distance the fluctuator moves across the junction. Recent studies (Martinis et al., 2005) point towards this coupling mechanism. Thus it is plausible that the fluctuators producing the charge noise in the charge qubits and the coherent fluctuators in the phase qubits are of the same origin. The former ones are located in the oxide away from the tunnel junction, while the latter ones reside in the oxide of the tunnel junction.

\section{Conclusions}

Josephson qubits have found their first application as sensitive meters of their environment. Measurements of qubit relaxation produced new surprising information about the properties of $1 / f$ noise. Motivated by these experiments, we have shown that an ensemble of coherent two-level systems with the distribution function, $P(\epsilon, \Delta) \propto \epsilon / \Delta$, produces Ohmic high-frequency noise and, at the same time, $1 / f$ low-frequency noise with strength which scales with temperature as $T^{2}$. The two branches of the noise power spectrum cross at $\omega \sim T$ in accordance with the experimental observation (Astafiev et al., 2004a). Thus, recent experimental findings (Astafiev et al., 2004a; Martinis et al., 2005) shed a new light on the nature of the low frequency fluctuations in mesoscopic systems.

\section{Acknowledgements}

This work was supported by the EU IST Project EuroSQIP and by the CFN (DFG), and the grant MD-2177.2005.2 (YM).

\section{References}

Aguado, R. and Kouwenhoven, L. P. (2000) Double Quantum Dots as Detectors of High-Frequency Quantum Noise in Mesoscopic Conductors, Phys. Rev. Lett. 84, 1986.

Anderson, P. W., Halperin, B. I., and Varma, C. M. (1972) Anomalous Low-Temperature Thermal Properties of Glasses and Spin Glasses, Phylos. Mag. 25, 1.

Astafiev, O. (2004), private communication.

Astafiev, O., Yu. A. Pashkin, Nakamura, Y., Yamamoto, T., and Tsai, J. S. (2004)a Quantum Noise in the Josephson Charge Qubit, Phys. Rev. Lett. 93, 267007.

Astafiev, O., Pashkin, Y. A., Yamamoto, T., Nakamura, Y., and Tsai, J. S. (2004)b Single-Shot Measurement of the Josephson Charge Qubit., Phys. Rev. B 69, 180507. 
Bernamont, J. (1937) Fluctuations de potentiel aux bornes d'un conducteur metallique de faible volume parcouru par un courant., Ann. Phys. (Leipzig) 7, 71.

Black, J. L. (1981) Low-Energy Excitations in Metallic Glasses, In H.-J. Güntherodt and H. Beck (eds.), Glassy metals, Berlin, Springer-Verlag.

Black, J. L. and Halperin, B. I. (1977) Spectral Diffusion, Phonon Echoes, and Saturation Recovery in Glasses at Low-Temperatures, Phys. Rev. B 16, 2879.

Bloch, F. (1957) Generalized Theory of Relaxation, Phys. Rev. 105, 1206.

Collin, E., Ithier, G., Aassime, A., Joyez, P., Vion, D., and Esteve, D. (2004) NMR-like Control of a Quantum Bit Superconducting Circuit., Phys. Rev. Lett. 93, 157005.

Cottet, A. (2002), PhD thesis, Université Paris VI.

de Sousa, R., Whaley, K. B., Wilhelm, F. K., and von Delft, J. (2005) Ohmic Noise from a Single Defect Center Hybridized with a Fermi Sea., Phys. Rev. Lett. 95, 247006.

Dutta, P. and Horn, P. M. (1981)a Low-Frequency Fluctuations in Solids, Rev. Mod. Phys. 53, 497.

Dutta, P. and Horn, P. M. (1981)b Low-Frequency Fluctuations in Solids: $1 / f$ Noise, Rev. Mod. Phys. 53, 497.

Esteve, D. and Vion, D. (2005) Solid State Quantum Bits, cond-mat/0505676.

Falci, G., D'Arrigo, A., Mastellone, A., and Paladino, E. (2005) Initial Decoherence in Solid State Qubits, Phys. Rev. Lett. 94, 167002.

Faoro, L., Bergli, J., Altshuler, B. A., and Galperin, Y. M. (2005) Models of Environment and $T_{1}$ Relaxation in Josephson Charge Qubits, Phys. Rev. Lett. 95, 046805.

Faoro, L. and Ioffe, L. B. (2005) Quantum Two Level Systems and Kondo-like Traps as Possible Sources of Decoherence in Superconducting Qubits., cond-mat/0510554.

Feng, S., Lee, P. A., and Stone, A. D. (1986) Sensitivity of the Conductance of a Disordered Metal to the Motion of a Single Atom: Implications for 1/f Noise, Phys. Rev. Lett. 56, 1960.

Galperin, Y. M., Altshuler, B. L., and Shantsev, D. V. (2004)a Low-Frequency Noise as a Source of Dephasing of a Qubit, In I. V. Lerner, B. L. Altshuler, and Y. Gefen (eds.), Fundamental Problems of Mesoscopic Physics, Dordrecht, Boston, London, Kluwer Academic Publishers, cond-mat/0312490.

Galperin, Y. M., Kozub, V. I., and Vinokur, V. M. (2004)b Low-Frequency Noise in Tunneling through a Single Spin, Phys. Rev. B 70, 033405.

Grishin, A., Yurkevich, I. V., and Lerner, I. V. (2005) Low-Temperature Decoherence of Qubit Coupled to Background Charges, Phys. Rev. B 72, 060509.

Imry, Y., Fukuyama, H., and Schwab, P. (1999) Low-Temperature Dephasing in Disordered Conductors: The Effect of "1/f" Fluctuations, Europhys. Lett. 47, 608.

Ithier, G., Collin, E., Joyez, P., Meeson, P. J., Vion, D., Esteve, D., Chiarello, F., Shnirman, A., Makhlin, Y., Schriefl, J., and Schön, G. (2005) Decoherence in a Superconducting Quantum Bit Circuit., Phys. Rev. B 72, 134519.

Kenyon, M., Lobb, C. J., and Wellstood, F. C. (2000) Temperature Dependence of Low-Frequency Noise in Al-A12O3-Al Single-Electron Transistors, J. Appl. Phys. 88, 6536.

Kogan, S. M. and Nagaev, K. E. (1984) On the Low-Frequency Current Noise in Metals, Solid State Comm. 49, 387.

Korotkov, A. N. and Averin, D. V. (2001) Continuous Weak Measurement of Quantum Coherent Oscillations, Phys. Rev. B 64, 165310.

Ludviksson, A., Kree, R., and Schmid, A. (1984) Low-Frequency 1/f Fluctuations of Resistivity in Disordered Metals, Phys. Rev. Lett. 52, 950.

Makhlin, Y. and Shnirman, A. (2004) Dephasing of Solid-State Qubits at Optimal Points, Phys. Rev. Lett. 92, 107001.

Martin, I., Bulaevskii, L., and Shnirman, A. (2005) Tunneling Spectroscopy of Two-level Systems Inside a Josephson Junction., Phys. Rev. Lett. 95, 127002. 
Martinis, J. M., Cooper, K. B., McDermott, R., Steffen, M., Ansmann, M., Osborn, K., Cicak, K., Oh, S., Pappas, D. P., Simmonds, R., and Yu, C. C. (2005) Decoherence in Josephson Qubits from Dielectric Loss., Phys. Rev. Lett. 95, 210503.

Nakamura, Y., Yu. A. Pashkin, Yamamoto, T., and Tsai, J. S. (2002) Charge Echo in a Cooper-Pair Box, Phys. Rev. Lett. 88, 047901.

Paladino, E., Faoro, L., Falci, G., and Fazio, R. (2002) Decoherence and 1/f noise in Josephson Qubits, Phys. Rev. Lett. 88, 228304.

Phillips, W. A. (1972) Tunneling States in Amorphous Solids, J. Low. Temp. Phys. 7, 351.

Rabenstein, K., Sverdlov, V. A., and Averin, D. V. (2004) Qubit Decoherence by Gaussian LowFrequency Noise, JETP Lett. 79, 783.

Redfield, A. G. (1957) On the theory of relaxation processes, IBM J. Res. Dev. 1, 19.

Schoelkopf, R. J., Clerk, A. A., Girvin, S. M., Lehnert, K. W., and Devoret, M. H. (2003) Qubits as Spectrometers of Quantum Noise, In Y. V. Nazarov (ed.), Quantum Noise in Mesoscopic Physics, Dordrecht, Boston, pp. 175-203, Kluwer Academic Publishers, cond-mat/0210247.

Schriefl, J. (2005), PhD Thesis, University of Karlsruhe.

Shnirman, A., Makhlin, Yu., and Schön, G. (2002) Noise and Decoherence in Quantum Two-Level Systems, Physica Scripta T102, 147.

Shnirman, A., Mozyrsky, D., and Martin, I. (2004) Output Spectrum of a Measuring Device at Arbitrary Voltage and temperature, Europhys. Lett. 67, 840.

Shnirman, A., Schön, G., Martin, I., and Makhlin, Y. (2005) Low- and High-Frequency Noise from Coherent Two-Level Systems, Phys. Rev. Lett. 94, 127002.

Siddiqi, I., Vijay, R., Pierre, F., Wilson, C. M., Metcalfe, M., Rigetti, C., Frunzio, L., and Devoret, M. H. (2004) RF-Driven Josephson Bifurcation Amplifier for Quantum Measurement, Phys. Rev. Lett. 93, 207002.

Simmonds, R. W., Lang, K. M., Hite, D. A., Nam, S., Pappas, D. P., and Martinis, J. M. (2004) Decoherence in Josephson Phase Qubits from Junction Resonators, Phys. Rev. Lett. 93, 077003.

VanHarlingen, D. J., Robertson, T. L., Plourde, B. L. T., Reichardt, P. A., Crane, T. A., and Clarke, J. (2004) Decoherence in Josephson-junction qubits due to critical current fluctuations, Phys. Rev. B 70, 064517.

Vion, D., Aassime, A., Cottet, A., Joyez, P., Pothier, H., Urbina, C., Esteve, D., and Devoret, M. H. (2002) Manipulating the quantum state of an electrical circuit, Science 296, 886.

Wallraff, A., Schuster, D. I., Blais, A., Frunzio1, L., Huang, R.-S., Majer, J., Kumar, S., Girvin, S. M., and Schoelkopf, R. J. (2004) Strong Coupling of a Single Photon to a Superconducting Qubit using Circuit Quantum Electrodynamics, Nature 431, 162.

Wellstood, F. C. (1988), PhD thesis, University of California, Berkeley.

Wellstood, F. C., Urbina, C., and Clarke, J. (2004) Flicker (1/f) Noise in the Critical Current of Josephson Junctions at 0.09-4.2 K, Appl. Phys. Lett. 85, 5296.

Wendin, G. and Shumeiko, V. S. (2005) Superconducting Quantum Circuits, Qubits and Computing, cond-mat/0508729.

Yamamoto, T., Pashkin, Y. A., Astafiev, O., Nakamura, Y., and Tsai, J. S. (2003) Demonstration of Conditional Gate Operation using Superconducting Charge Qubits., Nature 425, 941.

Zimmerli, G., Eiles, T. M., Kautz, R. L., and Martinis, J. M. (1992) Noise in the Coulomb Blockade Electrometer, Appl. Phys. Lett. 61, 237.

Zorin, A. B., Ahlers, F.-J., Niemeyer, J., Weimann, T., Wolf, H., Krupenin, V. A., and Lotkhov, S. V. (1996) Background Charge Noise in Metallic Single-Electron Tunneling Devices, Phys. Rev. B 53, 13682. 